\title{
Catalyst Evolution Enhances Production of Xylitol from Acetal- Stabilized Xylose
}

Ydna M. Questell-Santiago, Jher Hau Yeap, Masoud Talebi Amiri, Benjamin P. Le Monnier, and Jeremy S. Luterbacher*

Cite This: ACS Sustainable Chem. Eng. 2020, 8, 1709-1714

Read Online

\section{ACCESS | Llll Metrics \& More | 回 Article Recommendations | (s) Supporting Information}

ABSTRACT: We demonstrate a catalytic process for xylitol production based on the volatility and unique reactivity of diformylxylose (DFX), which can be produced at near theoretical yield from biomass and, contrary to xylose, can be easily purified by distillation. The apparent rate-limiting step was independent of hydrogen pressure, and catalytic studies showed a slow evolution of the $\mathrm{Pt} / \mathrm{C}$ catalyst that led to tripling of the xylitol yield. In-depth catalyst characterization attributed this activity increase to the formation of acidic carbon deposits, which created acid sites in close proximity to Pt. These proximal sites accelerated DFX hydrolysis while avoiding unfavorable isomerization reactions.

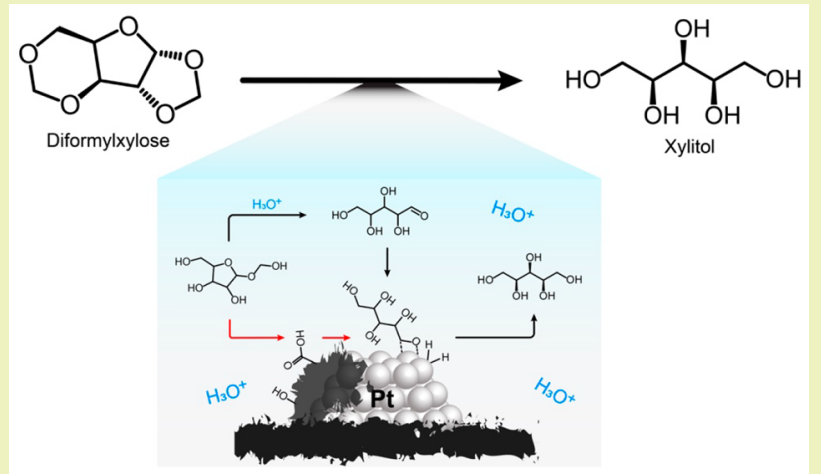

KEYWORDS: Xylitol, Diformylxylose, Lignocellulosic biomass, Platinum on activated carbon, XPS, DRIFT, TPD

\section{INTRODUCTION}

Xylitol has been identified as one of the top 12 sugar-based building block chemicals from biomass that has no petrochemical alternative by the United States Department of Energy (DOE). ${ }^{1}$ This polyol is commercially used as a natural sweetener in the food industry and in the pharmaceutical industry due to its insulin-independent metabolism and anticarcinogenic properties. ${ }^{2,3}$ It is also used in personal health products such as toothpaste and mouthwash and, more recently, has been proposed as a platform molecule for the production of alkanes, aromatics, propylene glycol, ethylene glycol, glycerol, xylaric acid, lactic acid, hydroxyfurans, and hydrogen. ${ }^{4,5}$ Given the numerous applications of xylitol, its production has increased more than 40 -fold in 40 years. ${ }^{4}$ In 2016, the global market for xylitol was estimated to be around 266,500 tonnes per year and is expected to overpass 1 billion USD by 2022. 4 Although currently produced from lignocellulose biomass (a relatively inexpensive and globally available raw material), ${ }^{5}$ its growth potential is limited by its costly manufacturing process notably due to the high energy consumption of the purification steps. ${ }^{2-4,7}$

Given the use of xylitol in food and pharmaceutical industries and given the sensitivity of hydrogenation catalysts, purity requirements generally demand that xylose hydrogenation occurs with a highly purified reactant. As xylose is nonvolatile and is generally mixed with other nonvolatile biomass-derived compounds including other sugars, it is typically separated by chromatography. This type of separation is costly in solvents and energy and represents a major process cost in xylitol production. ${ }^{7}$ Subsequently, the hydrogenation of xylose requires a hydrogen pressure around 40-100 bar to enhance the dissociation of hydrogen on the surface of the redox-active metal catalyst and maintain sufficient hydrogen coverage. ${ }^{4}$ The surface reaction between absorbed atomic hydrogen and absorbed xylose is the rate limiting step. The most common metals used for xylose hydrogenation are ruthenium $(\mathrm{Ru})$, rhodium $(\mathrm{Rh})$, nickel $(\mathrm{Ni})$, and palladium (Pd). ${ }^{2,4}$

Recently, we have shown that the addition of an aldehyde during an organosolv pretreatment can facilitate the recovery and upgrading of all three major fractions in biomass. ${ }^{8-10}$ This is due to the simultaneous stabilization of lignin fragments and xylose by the aldehydes that form acetal groups with available diol functionalities. These acetals notably prevent dehydration reactions, which are usually the first step toward lignin and sugar degradation reactions. ${ }^{11}$ When using formaldehyde as the stabilizing aldehyde, this pretreatment produces near quantitative yields of diformylxylose (DFX, formaldehyde-stabilized xylose) from hemicellulose and stabilized lignin while leaving behind a highly pure solid cellulose. Here, we propose to use

Received: October 29, 2019

Revised: December 9, 2019

Published: January 10, 2020 
DFX, which is volatile and can be easily purified by distillation, to produce xylitol. We notably demonstrate its ease of separation compared to xylose and characterize its yet unexplored reactivity.

\section{RESULTS AND DISCUSSION}

Biomass Pretreatment. Diformylxylose was produced from beech wood at a solids loading of 18 wt \% using a solution of water, gamma-valerolactone (GVL), formaldehyde (FA), and sulfuric acid in a $1 \mathrm{~L}$ Parr reactor equipped with a custom impeller. ${ }^{12}$ These pretreatment conditions led to a DFX yield and concentration of $86 \%$ and $3.5 \mathrm{wt} \%$, respectively (Table S1), with over $90 \%$ of cellulose recovery upon filtration. A lower biomass loading during pretreatment was able to produce a $98 \%$ DFX yield. ${ }^{8}$ Because the use of FA produces high-quality lignin, ${ }^{9}$ we explored its recovery and upgrading. After precipitation, the stabilized-lignin hydrogenolysis led to a $30 \%$ monomer yield ( $72 \%$ of what could be obtained by direct hydrogenolysis of the raw biomass, which can be considered as the maximal yield ${ }^{13}$ ). Interestingly, when using GVL and sulfuric acid during pretreatment, the resulting lignin led to a monomer product distribution with a $58 \%$ selectivity toward propylsyringol (Table S2), which is over twice what had been produced in the past when using alternate solvents with formaldehyde. This difference appeared to due to the reduced alkylation of ring positions by formaldehyde in GVL compared to other solvents.

Liquid $\mathrm{CO}_{2}$ extraction was used after precipitating stabilized lignin to remove contaminants from the pretreatment broth. This led to the recovery of $98 \%$ and $99 \%$ of the original GVL and DFX, respectively, in the liquid $\mathrm{CO}_{2}$ phase. After $\mathrm{CO}_{2}$ removal by depressurization, the resulting extracted solution contained 92 wt \% GVL and 4 wt \% DFX. A simplified overall process diagram is shown in Figure S1. The separation of DFX from GVL was done by distillation due to the volatility of both components. GVL distilled first, followed by DFX. After $8 \mathrm{~h}$ at $250{ }^{\circ} \mathrm{C}$, 9\% of DFX degraded, and $100 \%$ of the GVL was recovered. To limit this degradation, distillation was carried out under reduced pressure at conditions commonly used for oil refining. ${ }^{14}$ In these conditions, 99\% DFX and 100\% GVL were recovered using a two-step $\left(80\right.$ and $95{ }^{\circ} \mathrm{C}$ ) distillation process (Figure S1). The distillate from the first step at $80^{\circ} \mathrm{C}$ had a DFX concentration of 1 wt $\%$, which was then redistilled to mimic a multistage column. The bottoms were combined and used in the second step at $95{ }^{\circ} \mathrm{C}$ where DFX was recovered with a purity of $98 \%$. More details concerning these steps are available in Section 2 of the SI. We detail xylitol production below, but overall, this process demonstrated the potential to produce xylitol with a total yield from xylan in raw biomass of $60 \%-71 \%$ (dependent on the initial biomass loading, Figure S1).

Reaction Network of Tandem Hydrolysis-Hydrogenation of Diformylxylose to Xylitol. In the past, hydrogenation of xylose has been done at temperatures slightly above $100{ }^{\circ} \mathrm{C}$ to eliminate the issue of competitive adsorption between xylose and hydrogen. In our case, the conversion of DFX to xylitol was carried out at $185-200{ }^{\circ} \mathrm{C}$ to ensure that the water had the necessary concentration of protons to catalyze the removal of acetal functionalities from DFX. Past studies have found that platinum $(\mathrm{Pt})$ on carbon had a higher activity for the hydrogenation of xylose compared to $\mathrm{Ru}$ on carbon and Raney nickel. ${ }^{15}$ In this work, Liang and Jiang reported that $\mathrm{Pt}$ on carbon $(\mathrm{Pt} / \mathrm{C}$ ) could yield up to $97 \%$ xylitol from xylose at $120{ }^{\circ} \mathrm{C}$ and 55 bar $\mathrm{H}_{2}$ in batch reactions. ${ }^{15}$ Given these results, we decided to use $\mathrm{Pt} / \mathrm{C}$ as our catalyst. The catalyst preparation and characterization are detailed in Section 2 of the SI. After preparation, the catalyst had a Brunauer-Emmett-Teller (BET) surface area of 1002 $\mathrm{m}^{2} / \mathrm{g}$, a total metal loading of $2.6 \mathrm{wt} \%$, and a metal dispersion of $35 \%$ (Table S3).

Catalytic tests were done using DFX synthesized and purified from pure xylose following the detailed procedure given in Section 2 of the SI. Due to the quantity required for flow experiment, our lab scale facilities prevented the production of sufficient DFX to run these experiments with beech wood-derived product. The reaction was performed using a flow reactor at $185{ }^{\circ} \mathrm{C}$ with a catalyst bed of $0.50 \mathrm{~g}$ of $\mathrm{Pt} / \mathrm{C}$ and a hydrogen pressure of $20 \mathrm{bar}$ at a flow rate of 50 $\mathrm{mL} / \mathrm{min}$. At a weight hourly space velocity (WHSV) of 0.38 $\mathrm{h}^{-1}$, DFX reached a stable conversion of $\sim 90 \%$ after an induction period of $20 \mathrm{~h}$ (Figure 1).

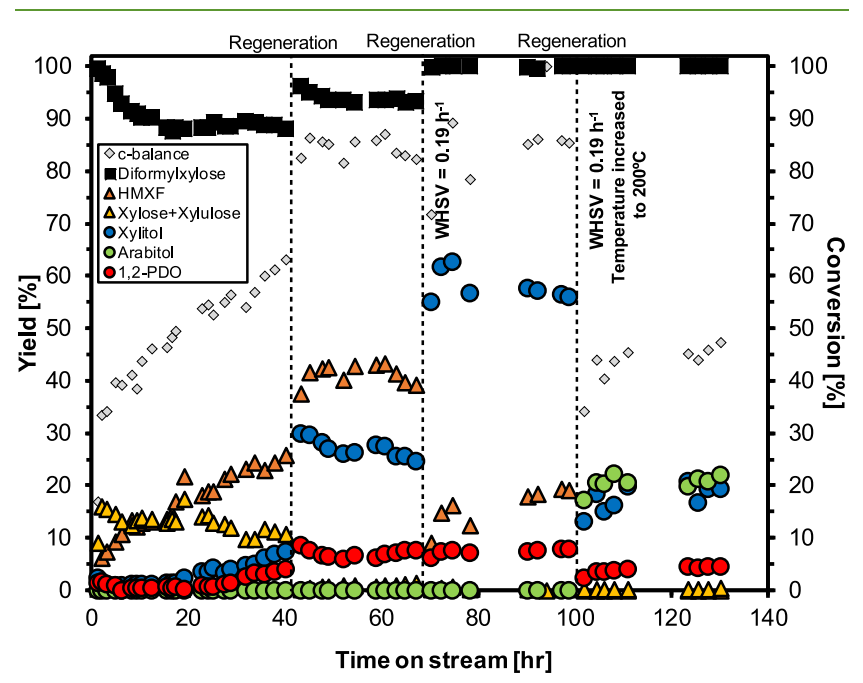

Figure 1. Upgrading of diformylxylose by tandem hydrolysishydrogenation over $\mathrm{Pt} / \mathrm{C}$ in the presence of hydrogen. (WHSV = $0.38 \mathrm{~h}^{-1}, \mathrm{H}_{2}$ flow $=50 \mathrm{~mL} / \mathrm{min}, P_{\mathrm{H} 2}=20 \mathrm{bar}, \mathrm{T}=185^{\circ} \mathrm{C}$, and feed $=$ 2 wt \% of DFX in water). WHSV was calculated based on DFX mass flow and total catalyst weight.

The principal intermediates that were identified were xylose and xylulose with a mole ratio of 0.82 xylose/xylulose. The intermediate 1-hydroxymethylxylofuranose (HMXF) was also detected (structure shown in Scheme 1; for full characterization, see our previous work ${ }^{8}$ ). Although HMXF appeared to be the main intermediate after a $20 \mathrm{~h}$ induction time, the HMXF concentration was estimated assuming the same response factor as DFX, which can lead to some uncertainty. Therefore, in the case of HMXF, we could only conclude that its formation was increasing over time. In general, the hydrolysis of DFX appeared to occur rapidly compared to the hydrolysis of HMXF and hydrogenation of xylose and xylulose. Based on these observations and the detection of other compounds such as 2-methylfuran (2-MF), 2-methyltetrahydrofuran (2-MTHF), and other over hydrogenated products in trace quantities, we proposed a reaction network of the conversion of DFX to polyols (Scheme 1).

Although the carbon balance increased over time suggesting a decrease in carbon deposits, the main increase in products was due to HMXF, while xylose and xylulose remained present, 
Scheme 1. Proposed Reaction Network for Conversion of Diformylxylose to Xylitol in Aqueous Media ${ }^{a}$

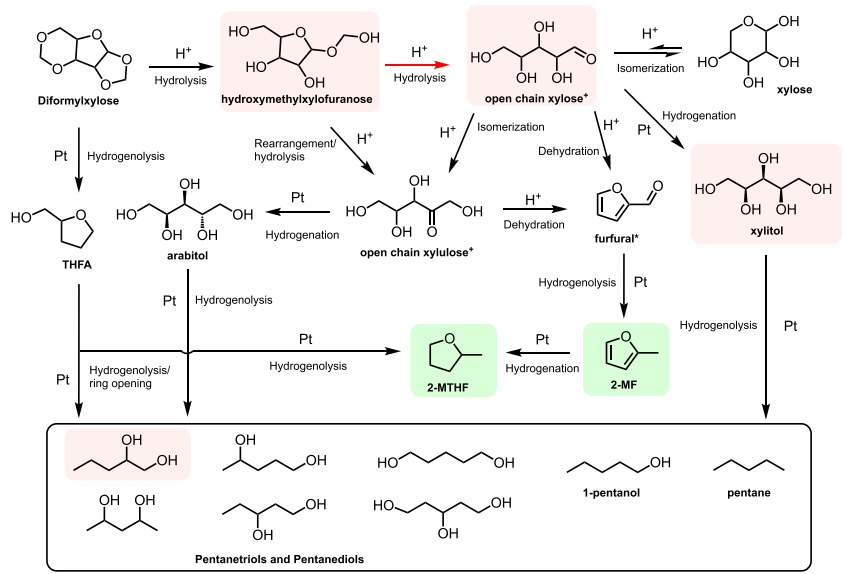

${ }^{a}$ Compounds were identified in either liquid or gas phases. The main products found in the aqueous phase are highlighted in pink. The main products found in the gas phase are highlighted in light green. Neither $\mathrm{CO}$ nor $\mathrm{CO}_{2}$ were detected in the GC using a TCD. The red arrow highlights the suggested rate-limiting step. $*$ : not detected. +: detected in their pyranose form.

all of which are nonhydrogenated molecules. The lack of a significant hydrogenation products could have been due to the blockage of $\mathrm{Pt}$ active sites by carbon deposits. After $40 \mathrm{~h}$ on stream, the catalyst was regenerated by reduction under $\mathrm{H}_{2}$ (details in Section 2 of the SI). After regeneration, we observed an increase in HMXF, as well as hydrogenation products and DFX conversion (Figure 1). In addition, xylose and xylulose were fully consumed. Although catalyst regeneration alone is unlikely to have fully removed carbon deposits, the aforementioned observations led us to conclude the following: (1) Regeneration created additional hydrogenation sites. (2) Within the presence of these sites, the rate-limiting step was the hydrolysis of HMXF and not the hydrogenation of pentoses. Therefore, after a second regeneration, WHSV was decreased to $0.19 \mathrm{~h}^{-1}$ in order to increase the retention time of intermediates (in this case, principally HMXF). These conditions led to a maximum yield of xylitol of 61\% (a 2fold increase) and a decrease in the HMXF yield of $25 \%$. Although HMXF yields should be interpreted qualitatively due to the aforementioned uncertainty in its quantification, its percentage decrease was independent of these quantification assumptions.

The reaction was also carried out at a higher temperature in order to increase the equilibrium concentration of protons in the water and thus increase the hydrolysis of HMXF. At 200 ${ }^{\circ} \mathrm{C}$, no HMXF was detected (Figure 1). However, the increase in temperature appeared to promote the reversible isomerization of xylose to xylulose leading to the formation of arabitol. Alternatively, the ring opening of HMXF could have also lead to the formation of xylulose. ${ }^{8}$ In any of these cases, the selectivity of the process decreases, which is what we observed.

Changes in Catalyst Active Sites. In order to understand the phenomenon that led to an increase in the quantity of hydrogenation products over $60 \mathrm{~h}$ time-on-stream and after catalyst regeneration, we studied the product distribution at the conditions with the highest xylitol yields $\left(185^{\circ} \mathrm{C}, 0.19 \mathrm{~h}^{-1}\right.$, 20 bar $\mathrm{H}_{2}$ ). The study was carried out over a fresh catalyst bed reduced in situ, i.e., after two thermal treatments. The highest hydrogenation activity of pentoses was observed after a forth catalyst reduction (Figure 2): The first reduction was after

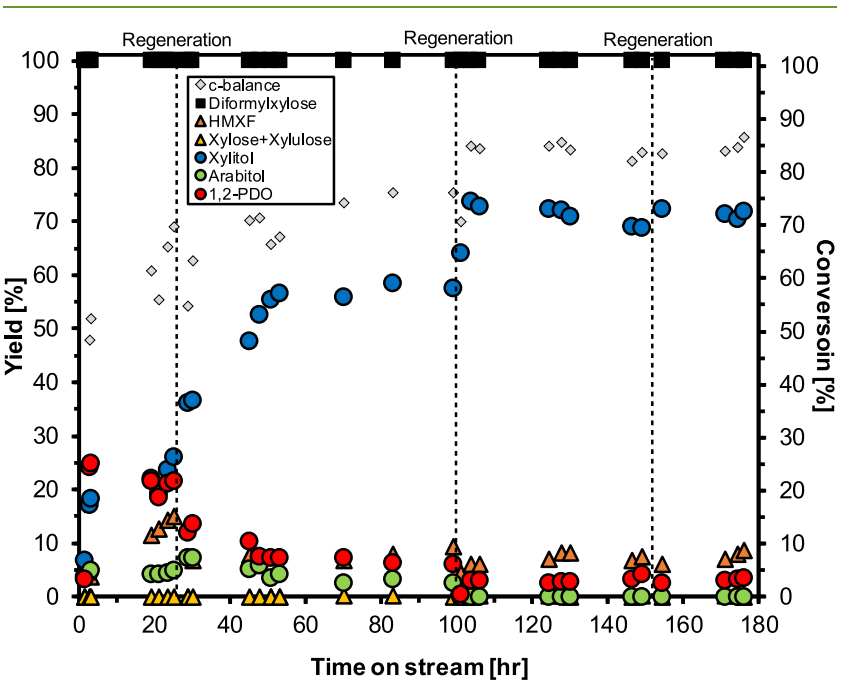

Figure 2. Changes in catalyst activity after multiple regenerations of the catalyst bed at 20 bar of $\mathrm{H}_{2}$. (WHSV $=0.19 \mathrm{~h}^{-1}, \mathrm{H}_{2}$ flow $=50$ $\mathrm{mL} / \mathrm{min}, T=185^{\circ} \mathrm{C}$, and feed $=2 \mathrm{wt} \%$ of DFX in water). WHSV was calculated based on DFX mass flow and total catalyst weight.

catalyst preparation (followed by passivation). The second reduction was performed in situ prior to the reaction. The third and forth reductions were performed during regenerations following the indicated amount of time-on-stream. At the beginning of the run, full conversion was observed, and only negligible quantities of pentoses were detected. For over $20 \mathrm{~h}$ of reaction, the main product was 1,2-pentanediol (1,2PDO), which then was slowly overtaken by xylitol. The formation of 1,2-PDO likely largely proceeded through ringopening/hydrogenation of tetrahydrofurfuryl alcohol (THFA, which was only detected when 1,2-PDO was the main product) and not through furfural, given that neither furfural nor furfuryl alcohol were detected at any reaction conditions (the most common route). ${ }^{16,17}$ The further hydrogenation of xylitol was not considered a major contributing route given the relatively low temperature used in this system compared to those used for diol production from xylitol over $\mathrm{Pt} / \mathrm{C},{ }^{18}$ the uncharacteristically high selectivity of 1,2-PDO compared to other triols and diols (which are usually produced in significant quantities when xylitol is the main reactant), and the low yield of all overhydrogenation products when xylitol is the main product (Figure 2, Figures S3a and S4). This suggested that during the induction period the hydrogenolysis of DFX was more favorable than hydrolysis. Arabitol was detected at a yield of about 5\%, indicating that there was limited formation of xylulose.

After the first regeneration, the yield of xylitol increased rapidly over $25 \mathrm{~h}$ and stabilized at $60 \%$ yield for about $50 \mathrm{~h}$. As the yield of xylitol increased, the formation of 1,2-PDO decreased, suggesting that the changes in catalytic activity favored the tandem hydrolysis-hydrogenation of DFX instead of the hydrogenolysis of DFX or the overhydrogenation of xylitol. A second regeneration led to a further xylitol yield increase of $15 \%$. The catalyst was reasonably stable for $50 \mathrm{~h}$ with only a small decrease in xylitol yield of 5\%. A third regeneration was performed, but no significant change in yield 
was observed. The yield of xylitol remained stable around $72 \%$ for $25 \mathrm{~h}$.

The same conditions were tested with 50 bar of hydrogen pressure (Figure S2). The evolution of xylitol and 1,2-PDO was different at the beginning of the reaction, but after the first regeneration, the performance was almost identical to the run at 20 bar of $\mathrm{H}_{2}$. The second regeneration increased the xylitol yield by $10 \%$, but the yield then rapidly decreased. This insensitivity to pressure, in combination with the observation of HMXF being the main intermediate (Figure 1), further pointed to the hydrolysis of HMXF (highlighted in red in Scheme 1) being the rate-limiting step for the production of xylitol. This mechanism provides opportunities to further lower the operational $\mathrm{H}_{2}$ pressure, but this would not guarantee that water remains liquid. Therefore, we will explore changing the $\mathrm{H}_{2}$ partial pressure in subsequent work.

To investigate just the effect of reduction, a fresh catalyst was reduced in situ for $9 \mathrm{~h}$ prior to reaction (originally $3 \mathrm{~h}$ ). This long reduction was done to mimic the total length of the four thermal treatments that were done prior to the point when the highest yield of xylitol (74\%) was obtained but without the influence of carbon deposits. However, these thermal treatments by themselves did not appear to be responsible for the increase in the catalyst activity. In fact, the catalyst was less active and selective compared to the catalyst bed reduced for only $3 \mathrm{~h}$ (Figure S3a). For over $30 \mathrm{~h}$, the yield of xylitol was less than $6 \%$, while the 1,2-PDO yield slightly increased to $13 \%$ and then decreased to $6 \%$. The gas phase data showed a maximum yield of $9 \%$ of $2-\mathrm{MF}$ and trace quantities of 2-MTHF (Figure S3b). No other compounds were detected. Further time-on-stream and regenerations led to similar changes in the catalyst activity with a delay of about $30 \mathrm{~h}$ featuring a similarly slow increase in the xylitol yield as that seen in Figure 2, which was also accompanied by increases in pressure drop (Figure S3b). Altogether, these data led us to conclude that the changes in activity are linked to support modifications or to the formation of carbon deposits in the vicinity of Pt nanoparticles.

To further explore these hypotheses, the spent catalyst resulting from the run at 20 bar of $\mathrm{H}_{2}$ (Figure 2) was characterized and showed a BET surface area and metal dispersion of $377 \mathrm{~m}^{2} / \mathrm{g}$ and $11 \%$, respectively (Table S3). The decrease in BET surface area, pore volume, pore area, and dispersion (without significant changes in particles size, see Figure S5) reconfirmed that carbon deposits had formed. The formation of carbon deposits has been known to affect catalyst's activity by chemically or physically adsorbing on metal surface sites, encapsulating metal particles and plugging micropores and mesopores. The latter effect can block the access to actives sites within these pores and reduce total surface area, both of which were observed during characterization. For these reasons, carbon deposits generally lead to catalyst deactivation by blocking active sites. In our system, we observe the opposite. Interestingly, ammonia temperatureprogrammed desorption ( $\mathrm{NH}_{3}$-TPD) showed a significant increase in total acid sites from 14.6 to $44.6 \mu \mathrm{mol} \mathrm{NH} / \mathrm{g}$ (Figure S6) between the fresh and spent catalysts. This increase was attributed to the formation of acidic functional groups on carbon deposits. These functional groups were identified and characterized by $\mathrm{CO}$ and $\mathrm{CO}_{2}$ TPD of the catalyst (Figure S7), diffuse reflectance infrared Fourier transform spectroscopy (DRIFT) (Figure 3), and X-ray photoelectron spectroscopy (XPS) (Figure $4 \mathrm{~b}$ and $\mathrm{d}$ ). We
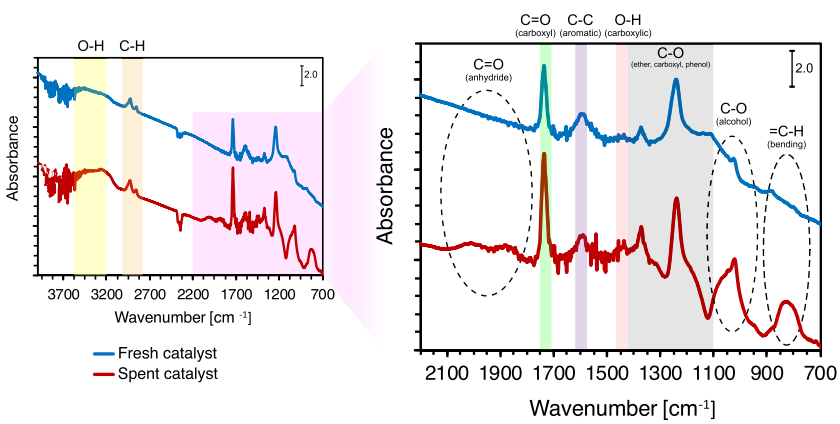

Figure 3. Diffuse reflectance infrared Fourier transform spectroscopy (DRIFT) of the fresh and spent $\mathrm{Pt} / \mathrm{C}$ catalyst. (The spent catalyst was used with WHSV $=0.19 \mathrm{~h}^{-1}$ at $20 \mathrm{bar} \mathrm{H}_{2}$ and $185^{\circ} \mathrm{C}$ for $180 \mathrm{~h}$, see Figure 2). Bands were assigned based on previous literature reports. $^{19-23}$

found that the abundance of carboxyl and hydroxyl groups on the spent catalyst increased compared to ether and carbonyl groups. The DRIFT spectra of the spent catalyst revealed the appearance of carboxylic anhydrides and hydroxyl groups, along with an increase in the carboxylic acid groups respective to aromatics (Figure 3). The binding energy of oxygen in the spent catalyst measured by XPS confirmed the results from the DRIFT spectra by showing an increase in the abundance of carboxyl and hydroxyl groups and a decrease in carbonyl groups compared to the fresh catalyst (Figure $4 \mathrm{~b}$ and $\mathrm{d}$ and Table S4). Interestingly, the XPS results also showed the appearance of metal oxide in the spent catalyst (discussed further below, Figure 4d).

To try to artificially mimic the formation of acid sites on the support only (as opposed to forming acidic carbon deposits directly on $\mathrm{Pt}$ nanoparticles), we prepared a functionalized activated carbon (nitric acid-treated activated carbon, details in Section 2 of the SI) with a total acid sites of $45.3 \mu \mathrm{mol} \mathrm{NH}_{3} / \mathrm{g}$, very similar to the spent catalyst (Table S3 and Figure S8b). When this material was used in catalytic test, we saw a rapid drop in conversion from $90 \%$ to $20 \%$ in $7 \mathrm{~h}$ (Figure S8a). This drop was accompanied by a significant increase in pressure drop (10 bar within the first $8 \mathrm{~h}$ ), while the samples that were taken were dark brown in color, which was not observed with samples from reactions with a nontreated activated carbon. All of these observations were an indication of sugar degradation and formation of carbon deposits. Interestingly, the use of a more acidic support did not initially increase the formation of xylitol but rather just increased carbon deposition. In contrast, after the formation of carbon deposits, the catalyst showed an increase in xylitol production, following a similar behavior as the experiment at 50 bar of $\mathrm{H}_{2}$ (Figure S2 and Figure S8a). Experiments run in batch with various acidic supports did not show a clear correlation with changes in the total amount of acid sites (Figure S9).

Therefore, a phenomenon beyond simple increases in support acidity appear to be taking place. X-ray powder diffraction (XRD) analyses did not show significant differences in crystalline structure nor mean crystallite size between the fresh and spent catalyst (Figure S10). At the same time, XPS revealed the presences of $\mathrm{PtO}$ and $\mathrm{Pt}(\mathrm{OH})_{2}$, confirming the increase in metal oxide signals for oxygen in the spent catalyst compared to the fresh catalyst (Figure 4a to d, Table S4). Both catalysts were analyzed without being exposed to air after reduction under $\mathrm{H}_{2}$ flow at $300{ }^{\circ} \mathrm{C}$. This suggested that carbon deposits were growing on top of the nanoparticle which 
a

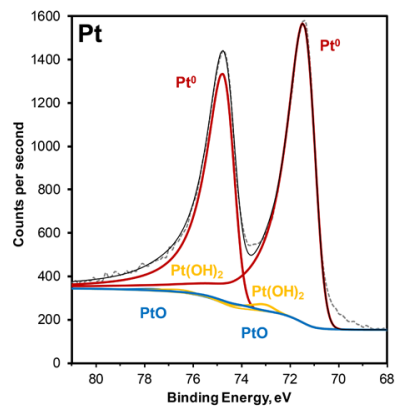

C

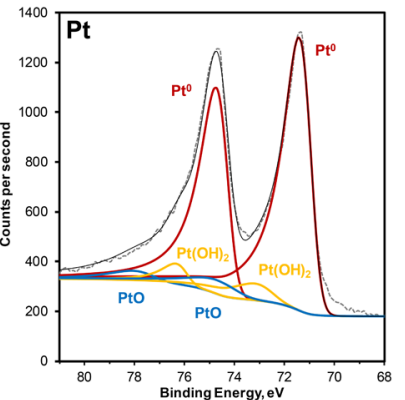

b

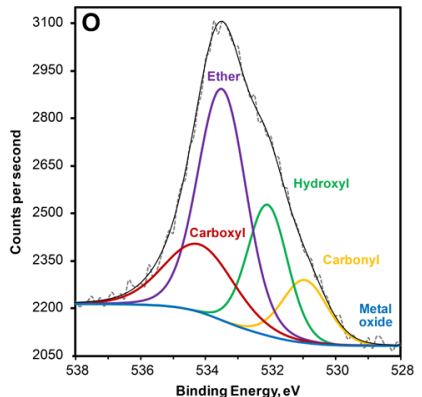

d

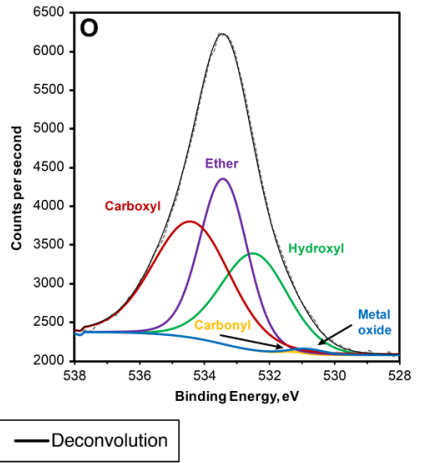

e

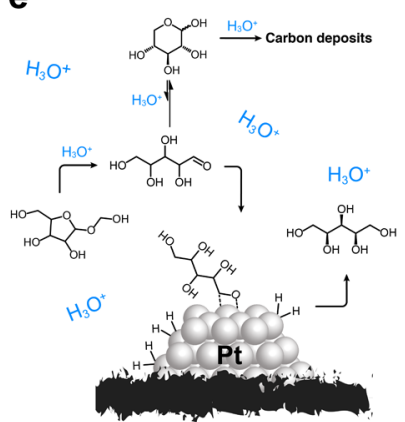

f

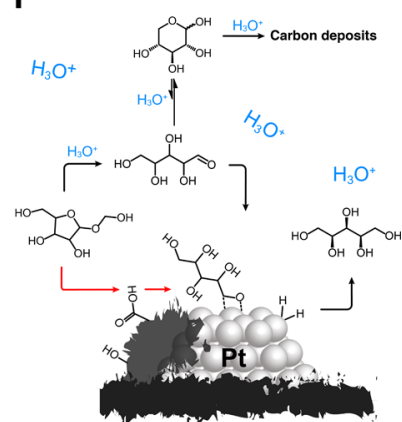

Figure 4. X-ray photoelectron spectroscopy (XPS) of the (a and b) fresh and ( $c$ and $d$ ) spent $\mathrm{Pt} / \mathrm{C}$ catalyst and the schematic representation of the role of carbon deposits and their acidic functional groups in the production of xylitol from diformylxylose. (The spent catalyst was used with WHSV $=0.19 \mathrm{~h}^{-1}$ at $20 \mathrm{bar} \mathrm{H}_{2}$ and $185^{\circ} \mathrm{C}$ for $180 \mathrm{~h}$, see Figure 2). The proposed reaction pathway of HMXF to xylitol using a (e) fresh and (f) spent Pt/C catalyst. Functional groups were assigned based on past literature reports using the binding energy of oxygen. ${ }^{19,24}$

prevented the complete reduction of Pt. Given that HMXF is the main intermediate and no pentoses were detected, we propose that the acid-functionalized carbon deposits could increase the hydrolysis of HMXF. Because this, hydrolysis takes place on acid sites near $\mathrm{Pt}^{0}$ atoms, and the open chain of xylose could rapidly adsorb and react, minimizing its chance to isomerize to xylopyranose (Figure 4f). This isomerization limits the reaction rate by removing the open chain xylose through a reversible reaction, reducing the presence of the precursor to xylitol (Figure 4e). Indeed, when the acid sites were farther away from the Pt particle (as was the case for the experiment with a functionalized carbon support and other acidic supports), we observed the significant aforementioned increase in carbon deposits formation by subsequent reactions catalyzed by acid sites, which is more likely to occur when no hydrogenation site is close by.

\section{CONCLUSION}

In conclusion, we have described an unexplored process to produce xylitol from raw beech wood using DFX as an intermediate. Even though several steps could be further optimized, we show that, due to the properties of DFX and notably its volatility, this molecule can be easily purified by distillation, which avoided costly processes like the use of activated carbon, ion exchange chromatography, or other sophisticated purification methods. In addition, the process is compatible with the valorization of the three main lignocellulosic biomass fractions, as the resulting formaldehyde-stabilized lignin could be separately upgraded to monomers at yields above $30 \%$. The upgrading of DFX to xylitol could be performed through a tandem hydrolysishydrogenation reaction where the hydrolysis of HMXF (produced by the partial deprotection of DFX) seems to be the rate-limiting step. This mechanism allowed us to run xylitol production at somewhat lower $\mathrm{H}_{2}$ pressures than those that are typically used for xylose hydrogenation (20 vs 40-100 bar). Finally, we found that, over the course of the reaction, the active sites of the catalyst changed, which led to progressive increases in xylitol yields. XPS studies on the spent catalyst suggested that this is due to the formation of acidic carbon deposits on top of Pt nanoparticles. These acidic functional groups on carbon deposits accelerated the rate-limiting step by hydrolyzing HMXF, but due to its proximity to $\mathrm{Pt}^{0}$, the isomerization to xylopyranose was avoided by the adsorption of the open chain of xylose. Overall, these results demonstrate that aldehyde functionalized xylose can, due to its unique properties and reactivity, open new conversion routes and process possibilities in the context of biorefineries.

\section{ASSOCIATED CONTENT}

\section{Supporting Information}

The Supporting Information is available free of charge at https://pubs.acs.org/doi/10.1021/acssuschemeng.9b06456.

Chemicals and materials, experimental procedure for biomass pretreatment, lignin precipitation, diformylxylose purification, catalyst preparation and characterization, TPD, XRD, XPS, and supporting tables and figures (PDF)

\section{AUTHOR INFORMATION}

\section{Corresponding Author}

Jeremy S. Luterbacher - Ecole Polytechnique Federale de Lausanne (EPFL), Lausanne, Switzerland; (1) orcid.org/ 0000-0002-0967-0583; Email: jeremy.luterbacher@ epfl.ch

\section{Other Authors}

Ydna M. Questell-Santiago - Ecole Polytechnique Féderale de Lausanne (EPFL), Lausanne, Switzerland; (1) orcid.org/0000-0002-6447-9263

Jher Hau Yeap - Ecole Polytechnique Féderale de Lausanne (EPFL), Lausanne, Switzerland; (1) orcid.org/ 0000-0002-4954-4688 
Masoud Talebi Amiri - École Polytechnique Federale de Lausanne (EPFL), Lausanne, Switzerland; 이이.org/ 0000-0003-4180-3769

Benjamin P. Le Monnier - Ecole Polytechnique Federale de Lausanne (EPFL), Lausanne, Switzerland

Complete contact information is available at:

https://pubs.acs.org/10.1021/acssuschemeng.9b06456

\section{Notes}

The authors declare no competing financial interest.

\section{ACKNOWLEDGMENTS}

This work was supported by the Swiss National Science Foundation through Grant PYAPP2_154281, by the Swiss Competence Center for Energy Research: Biomass for a Swiss Energy Future through the Swiss Commission for Technology and Innovation Grant KTI.2014.0116, by the European Research Council (ERC) under the European Union's Horizon 2020 research and innovation program (Starting Grant CATACOAT, No. 758653), and by EPFL. The authors thank M. Studer from the Bern University of Applied Sciences (Switzerland) for providing beech wood.

\section{REFERENCES}

(1) Werpy, T.; Petersen, G. Top Value Added Chemicals from Biomass: Vol. I - Results of Screening for Potential Candidates from Sugars and Synthesis Gas; DOE/GO-102004-1992; National Renewable Energy Lab., Golden, CO, USA, 2004. DOI: 10.2172/15008859.

(2) Rafiqul, I. S. M.; Sakinah, A. M. M. Processes for the Production of Xylitol-A Review. Food Rev. Int. 2013, 29 (2), 127-156.

(3) Dasgupta, D.; Bandhu, S.; Adhikari, D. K.; Ghosh, D. Challenges and Prospects of Xylitol Production with Whole Cell Bio-Catalysis: A Review. Microbiol. Res. 2017, 197, 9-21.

(4) Delgado Arcaño, Y.; Valmaña García, O. D.; Mandelli, D.; Carvalho, W. A.; Magalhães Pontes, L. A. Xylitol: A Review on the Progress and Challenges of Its Production by Chemical Route. Catal. Today 2018, na DOI: 10.1016/j.cattod.2018.07.060.

(5) Luterbacher, J. S.; Martin Alonso, D.; Dumesic, J. A. Targeted Chemical Upgrading of Lignocellulosic Biomass to Platform Molecules. Green Chem. 2014, 16 (12), 4816-4838.

(6) Özüdoğru, H. M. R.; Nieder-Heitmann, M.; Haigh, K. F.; Görgens, J. F. Techno-Economic Analysis of Product Biorefineries Utilizing Sugarcane Lignocelluloses: Xylitol, Citric Acid and Glutamic Acid Scenarios Annexed to Sugar Mills with Electricity CoProduction. Ind. Crops Prod. 2019, 133, 259-268.

(7) Ravella, S. R.; Gallagher, J.; Fish, S.; Prakasham, R. S. Overview on Commercial Production of Xylitol, Economic Analysis and Market Trends. In D-Xylitol: Fermentative Production, Application and Commercialization; da Silva, S. S., Chandel, A. K., Eds.; Springer: Berlin, Heidelberg, 2012; pp 291-306. DOI: 10.1007/978-3-64231887-0 13.

(8) Questell-Santiago, Y. M.; Zambrano-Varela, R.; Talebi Amiri, M.; Luterbacher, J. S. Carbohydrate Stabilization Extends the Kinetic Limits of Chemical Polysaccharide Depolymerization. Nat. Chem. 2018, 10 (12), 1222.

(9) Shuai, L.; Amiri, M. T.; Questell-Santiago, Y. M.; Héroguel, F.; Li, Y.; Kim, H.; Meilan, R.; Chapple, C.; Ralph, J.; Luterbacher, J. S. Formaldehyde Stabilization Facilitates Lignin Monomer Production during Biomass Depolymerization. Science 2016, 354 (6310), 329333.

(10) Lan, W.; Amiri, M. T.; Hunston, C. M.; Luterbacher, J. S. Protection Group Effects During $\alpha, \gamma$-Diol Lignin Stabilization Promote High-Selectivity Monomer Production. Angew. Chem., Int. Ed. 2018, 57 (5), 1356-1360.

(11) Lan, W.; Luterbacher, J. Preventing Lignin Condensation to Facilitate Aromatic Monomer Production. Chimia 2019, 73, 591.
(12) Klingenberg, D. J.; Root, T. W.; Scott, C. T.; Houtman, C.; Bourne, K. J.; Subramaniam, V. High Temperature Rheometry of Lignocellulosic Biomass; The Society of Rheology 86th Annual Meeting, Philadelphia, PA, USA, October 6-9, 2014.

(13) Talebi Amiri, M.; Dick, G. R.; Questell-Santiago, Y. M.; Luterbacher, J. S. Fractionation of Lignocellulosic Biomass to Produce Uncondensed Aldehyde-Stabilized Lignin. Nat. Protoc. 2019, 14, 921-954.

(14) Vacuum Distillation Is a Key Part of the Petroleum Refining Process, 2012. Today in Energy. U.S. Energy Information Administration (EIA) https://www.eia.gov/todayinenergy/detail. php?id=9130\# (accessed April 11, 2019).

(15) Liang, X.; Jiang, C. Atomic Layer Deposited Highly Dispersed Platinum Nanoparticles Supported on Non-Functionalized Multiwalled Carbon Nanotubes for the Hydrogenation of Xylose to Xylitol. J. Nanopart. Res. 2013, 15 (9), 1890.

(16) Gupta, K.; Rai, R. K.; Singh, S. K. Metal Catalysts for the Efficient Transformation of Biomass-Derived HMF and Furfural to Value Added Chemicals. Chem CatChem 2018, 10 (11), 2326-2349.

(17) Nakagawa, Y.; Tamura, M.; Tomishige, K. Catalytic Reduction of Biomass-Derived Furanic Compounds with Hydrogen. ACS Catal. 2013, 3 (12), 2655-2668.

(18) Kirilin, A. V.; Hasse, B.; Tokarev, A. V.; Kustov, L. M.; Baeva, G. N.; Bragina, G. O.; Stakheev, A. Y.; Rautio, A.-R.; Salmi, T.; Etzold, B. J. M.; Mikkola, J.-P.; Murzin, D. Y.; et al. Aqueous-Phase Reforming of Xylitol over Pt/C and Pt/TiC-CDC Catalysts: Catalyst Characterization and Catalytic Performance. Catal. Sci. Technol. 2014, 4 (2), 387-401.

(19) Figueiredo, J. L.; Pereira, M. F. R.; Freitas, M. M. A.; Órfão, J. J. M. Modification of the Surface Chemistry of Activated Carbons. Carbon 1999, 37 (9), 1379-1389.

(20) Dandekar, A.; Baker, R. T. K.; Vannice, M. A. Characterization of Activated Carbon, Graphitized Carbon Fibers and Synthetic Diamond Powder Using TPD and DRIFTS. Carbon 1998, 36 (12), 1821-1831.

(21) Chen, J. P.; Wu. Acid/Base-Treated Activated Carbons: Characterization of Functional Groups and Metal Adsorptive Properties. Langmuir 2004, 20 (6), 2233-2242.

(22) Shafeeyan, M. S.; Daud, W. M. A. W.; Houshmand, A.; Shamiri, A. A Review on Surface Modification of Activated Carbon for Carbon Dioxide Adsorption. J. Anal. Appl. Pyrolysis 2010, 89 (2), 143-151.

(23) Afonso, J. C.; Schmal, M.; Fréty, R. The Chemistry of Coke Deposits Formed on a Pt $\square$ Sn Catalyst during Dehydrogenation of NAlkanes to Mono-Olefins. Fuel Process. Technol. 1994, 41 (1), 13-25.

(24) Wan, X.; Zhou, C.; Chen, J.; Deng, W.; Zhang, Q.; Yang, Y.; Wang, Y. Base-Free Aerobic Oxidation of 5-Hydroxymethyl-Furfural to 2,5-Furandicarboxylic Acid in Water Catalyzed by Functionalized Carbon Nanotube-Supported Au-Pd Alloy Nanoparticles. ACS Catal. 2014, 4 (7), 2175-2185. 